\title{
Sharp Cusa and Becker-Stark inequalities
}

\author{
Chao-Ping Chen ${ }^{1 *}$ and Wing-Sum Cheung ${ }^{2}$
}

\footnotetext{
* Correspondence:

chenchaoping@sohu.com

'School of Mathematics and

Informatics, Henan Polytechnic,

University, Jiaozuo City 454003,

Henan Province, People's Republic

of China

Full list of author information is

available at the end of the article
}

\footnotetext{
Abstract

We determine the best possible constants $\theta, \bigotimes, \alpha$ and $\beta$ such that the inequalities

$$
\left(\frac{2+\cos x}{3}\right)^{\theta}<\frac{\sin x}{x}<\left(\frac{2+\cos x}{3}\right)^{\vartheta}
$$

and

$$
\left(\frac{\pi^{2}}{\pi^{2}-4 x^{2}}\right)^{\alpha}<\frac{\tan x}{x}<\left(\frac{\pi^{2}}{\pi^{2}-4 x^{2}}\right)^{\beta}
$$

are valid for $0<x<\pi / 2$. Our results sharpen inequalities presented by Cusa, Becker and Stark.

Mathematics Subject Classification (2000): 26D05.

Keywords: Inequalities, trigonometric functions
}

\section{Introduction}

For $0<x<\pi / 2$, it is known in the literature that

$$
\frac{\sin x}{x}<\frac{2+\cos x}{3} \text {. }
$$

Inequality (1) was first mentioned by the German philosopher and theologian Nicolaus de Cusa (1401-1464), by a geometrical method. A rigorous proof of inequality (1) was given by Huygens [1], who used (1) to estimate the number $\pi$. The inequality is now known as Cusa's inequality [2-5]. Further interesting historical facts about the inequality (1) can be found in [2].

It is the first aim of present paper to establish sharp Cusa's inequality.

Theorem 1. For $0<x<\pi / 2$,

$$
\left(\frac{2+\cos x}{3}\right)^{\theta}<\frac{\sin x}{x}<\left(\frac{2+\cos x}{3}\right)^{\vartheta}
$$

with the best possible constants

$$
\theta=\frac{\ln (\pi / 2)}{\ln (3 / 2)}=1.11373998 \ldots \text { and } \vartheta=1 \text {. }
$$


Becker and Stark [6] obtained the inequalities

$$
\frac{8}{\pi^{2}-4 x^{2}}<\frac{\tan x}{x}<\frac{\pi^{2}}{\pi^{2}-4 x^{2}} \quad\left(0<x<\frac{\pi}{2}\right) .
$$

The constant 8 and $\pi^{2}$ are the best possible.

Zhu and Hua [7] established a general refinement of the Becker-Stark inequalities by using the power series expansion of the tangent function via Bernoulli numbers and the property of a function involving Riemann's zeta one. Zhu [8] extended the tangent function to Bessel functions.

It is the second aim of present paper to establish sharp Becker-Stark inequality.

Theorem 2. For $0<x<\pi / 2$,

$$
\left(\frac{\pi^{2}}{\pi^{2}-4 x^{2}}\right)^{\alpha}<\frac{\tan x}{x}<\left(\frac{\pi^{2}}{\pi^{2}-4 x^{2}}\right)^{\beta}
$$

with the best possible constants

$$
\alpha=\frac{\pi^{2}}{12}=0.822467033 \ldots \text { and } \beta=1 .
$$

Remark 1. There is no strict comparison between the two lower bounds $\frac{8}{\pi^{2}-4 x^{2}}$ and $\left(\frac{\pi^{2}}{\pi^{2}-4 x^{2}}\right)^{\pi^{2} / 12}$ in (3) and (4).

The following lemma is needed in our present investigation.

Lemma 1 ([9-11]). Let $-\infty<a<b<\infty$, and $f, g:[a, b] \rightarrow \mathbb{R}$ be continuous on $[a, b]$ and differentiable in $(a, b)$. Suppose $g^{\prime} \neq 0$ on $(a ; b)$. If $f(x) / g^{\prime}(x)$ is increasing (decreasing) on $(a, b)$, then so are

$$
[f(x)-f(a)] /[g(x)-g(a)] \text { and }[f(x)-f(b)] /[g(x)-g(b)] .
$$

If $f^{\prime}(x)=g^{\prime}(x)$ is strictly monotone, then the monotonicity in the conclusion is also strict.

\section{Proofs of Theorems 1 and 2}

Proof of Theorem [1]. Consider the function $f(x)$ defined by

$$
\begin{aligned}
& F(x)=\frac{\ln \left(\frac{\sin x}{x}\right)}{\ln \left(\frac{2+\cos x}{3}\right)}, \quad 0<x<\frac{\pi}{2}, \\
& F(0)=1 \text { and } F\left(\frac{\pi}{2}\right)=\frac{\ln (\pi / 2)}{\ln (3 / 2)} .
\end{aligned}
$$

For $0<x<\pi / 2$, let

$$
F_{1}(x)=\ln \left(\frac{\sin x}{x}\right) \quad \text { and } \quad F_{2}(x)=\ln \left(\frac{2+\cos x}{3}\right) .
$$


Then,

$$
\frac{F_{1}^{\prime}(x)}{F^{\prime}{ }_{2}(x)}=\frac{-2 x \cos x-x \cos ^{2} x+2 \sin x+\sin x \cos x}{x \sin ^{2} x}=\frac{F_{3}(x)}{F_{4}(x)}
$$

where

$$
F_{3}(x)=-2 x \cos x-x \cos ^{2} x+2 \sin x+\sin x \cos x \quad \text { and } \quad F_{4}(x)=x \sin ^{2} x
$$

Differentiating with respect to $x$ yields

$$
\frac{F^{\prime}{ }_{3}(x)}{F^{\prime}{ }_{4}(x)}=\frac{2 x+2 x \cos x-\sin x}{\sin x+2 x \cos x} \triangleq F_{5}(x) \text {. }
$$

Elementary calculations reveal that

$$
F_{5}^{\prime}(x)=\frac{2 F_{6}(x)}{2 x \sin (2 x)+4 x^{2} \cos ^{2} x+\sin ^{2} x}
$$

where

$$
F_{6}(x)=\sin (2 x)+\left(2 x^{2}+1\right) \sin x-2 x-x \cos x .
$$

By using the power series expansions of sine and cosine functions, we find that

$$
F_{6}(x)=x^{3}-\frac{1}{10} x^{5}-\frac{19}{2520} x^{7}+2 \sum_{n=4}^{\infty}(-1)^{n} u_{n}(x)
$$

where

$$
u_{n}(x)=\frac{4^{n}-4 n^{2}-3 n}{(2 n+1) !} x^{2 n+1}
$$

Elementary calculations reveal that, for $0<x<\pi / 2$ and $n \geq 4$,

$$
\begin{aligned}
\frac{u_{n+1}(x)}{u_{n}(x)} & =\frac{x^{2}}{2} \frac{2^{2 n+2}-4 n^{2}-11 n-7}{(n+1)(2 n+3)\left(4^{n}-4 n^{2}-3 n\right)} \\
& <\frac{1}{2}\left(\frac{\pi}{2}\right)^{2} \frac{2^{2 n+2}-4 n^{2}-11 n-7}{(n+1)(2 n+3)\left(4^{n}-4 n^{2}-3 n\right)} \\
& =\frac{\pi^{2}}{8(n+1)} \frac{4^{n+1}-4 n^{2}-11 n-7}{(2 n+3)\left(4^{n}-4 n^{2}-3 n\right)} \\
& <\frac{\pi^{2}}{8(n+1)}<1 .
\end{aligned}
$$

Hence, for fixed $x \in(0, \pi / 2)$, the sequence $n \mapsto u_{n}(x)$ is strictly decreasing with regard to $n \geq 4$. Hence, for $0<x<\pi / 2$,

$$
F_{6}(x)=x^{3}-\frac{1}{10} x^{5}-\frac{19}{2520} x^{7}>0 \quad\left(0<x<\frac{\pi}{2}\right),
$$

and therefore, the functions $F_{5}(x)$ and $\frac{F_{3}^{\prime}(x)}{F_{4}^{\prime}(x)}$ are both strictly increasing on $(0, \pi / 2)$. 
By Lemma 1, the function

$$
\frac{F_{1}^{\prime}(x)}{F_{2}^{\prime}(x)}=\frac{F_{3}(x)}{F_{4}(x)}=\frac{F_{3}(x)-F_{3}(0)}{F_{4}(x)-F_{4}(0)}
$$

is strictly increasing on $(0, \pi / 2)$. By Lemma 1 , the function

$$
F(x)=\frac{F_{1}(x)}{F_{2}(x)}=\frac{F_{1}(x)-F_{1}(0)}{F_{2}(x)-F(0)}
$$

is strictly increasing on $(0, \pi / 2)$, and we have

$$
1=F(0)<F(x)=\frac{\ln \left(\frac{\sin x}{x}\right)}{\ln \left(\frac{2+\cos x}{3}\right)}<F\left(\frac{\pi}{2}\right)=\frac{\ln (\pi / 2)}{\ln (3 / 2)} \quad \forall x \in\left(0, \frac{\pi}{2}\right) .
$$

By rearranging terms in the last expression, Theorem 1 follows.

Proof of Theorem 2. Consider the function $f(x)$ defined by

$$
\begin{aligned}
& f(x)=\frac{\ln \left(\frac{\tan x}{x}\right)}{\ln \left(\frac{\pi^{2}}{\pi^{2}-4 x^{2}}\right)}, \quad 0<x<\frac{\pi}{2} \\
& f(0)=\frac{\pi^{2}}{12} \text { and } f\left(\frac{\pi}{2}\right)=1 .
\end{aligned}
$$

For $0<x<\pi / 2$, let

$$
f_{1}(x)=\ln \left(\frac{\tan x}{x}\right) \text { and } f_{2}(x)=\ln \left(\frac{\pi^{2}}{\pi^{2}-4 x^{2}}\right) .
$$

Then,

$$
\frac{f_{1}^{\prime}(x)}{f^{\prime}{ }_{2}(x)}=\frac{\left(\pi^{2}-4 x^{2}\right)(2 x-\sin (2 x))}{8 x^{2} \sin (2 x)} \triangleq g(x) .
$$

Elementary calculations reveal that

$$
4 x^{3} \sin ^{2}(2 x) g^{\prime}(x)=-\left(\pi^{2}+4 x^{2}\right) x \sin (2 x)-2\left(\pi^{2}-4 x^{2}\right) x^{2} \cos (2 x)+\pi^{2} \sin ^{2}(2 x) \triangleq h(x) .
$$

Motivated by the investigations in [12], we are in a position to prove $h(x)>0$ for $x \in$ $(0, \pi / 2)$.Let

$$
H(x)= \begin{cases}\lambda, & x=0 \\ \frac{h(x)}{x^{6}\left(\frac{\pi}{2}-x\right)^{2}} & 0<x<\frac{\pi}{2} \\ \mu, & x=\frac{\pi}{2}\end{cases}
$$

Where $\lambda$ and $\mu$ are constants determined with limits:

$$
\begin{aligned}
& \lambda=\lim _{x \rightarrow 0^{+}} \frac{h(x)}{x^{6}\left(\frac{\pi}{2}-x\right)^{2}}=\frac{224 \pi^{2}-1920}{45 \pi^{2}}=0.654740609 \ldots \\
& \mu=\lim _{t \rightarrow(\pi / 2)^{-}} \frac{h(x)}{x^{6}\left(\frac{\pi}{2}-x\right)^{2}}=\frac{128}{\pi^{4}}=1.31404572 \ldots
\end{aligned}
$$


Using Maple, we determine Taylor approximation for the function $H(x)$ by the polynomial of the first order:

$$
P_{1}(x)=\frac{32\left(7 \pi^{2}-60\right)}{45 \pi^{2}}+\frac{128\left(7 \pi^{2}-60\right)}{45 \pi^{3}} x
$$

which has a bound of absolute error

$$
\varepsilon_{1}=\frac{-1920-1920 \pi^{2}+224 \pi^{4}}{15 \pi^{4}}=0.650176097 \ldots
$$

for values $x \in[0, \pi / 2]$. It is true that

$$
H(x)-\left(P_{1}(x)-\mathcal{E}_{1}\right) \geq 0, \quad P_{1}(x)-\mathcal{E}_{1}=\frac{64\left(60 \pi^{2}+90-7 \pi^{4}\right)}{45 \pi^{4}}+\frac{128\left(7 \pi^{2}-60\right)}{45 \pi^{3}} x>0
$$

for $x \in[0, \pi / 2]$. Hence, for $x \in[0, \pi / 2]$, it is true that $H(x)>0$ and, therefore, $h(x)$ $>0$ and $g^{\prime}(x)>0$ for $x \in[0, \pi / 2]$. Therefore, the function $\frac{f_{1}^{\prime}(x)}{f_{2}^{\prime}(x)}$ is strictly increasing on. $(0, \pi / 2)$.By Lemma 1 , the function

$$
f(x)=\frac{f_{1}(x)}{f_{2}(x)}
$$

is strictly increasing on $(0, \pi / 2)$, and we have

$$
\frac{\pi^{2}}{12}=f(0)<f(x)=\frac{\ln \left(\frac{\tan x}{x}\right)}{\ln \left(\frac{\pi^{2}}{\pi^{2}-4 x^{2}}\right)}<f\left(\frac{\pi}{2}\right)=1 .
$$

By rearranging terms in the last expression, Theorem 2 follows.

\section{Acknowledgements}

Research is supported in part by the Research Grants Council of the Hong Kong SAR, Project No. HKU7016/07P.

\section{Author details}

${ }^{1}$ School of Mathematics and Informatics, Henan Polytechnic, University, Jiaozuo City 454003, Henan Province, People's Republic of China ${ }^{2}$ Department of Mathematics, the University of Hong Kong, Pokfulam Road, Hong Kong, China

\section{Authors' contributions}

All authors read and approved the final manuscript

\section{Competing interests}

The authors declare that they have no competing interests.

Received: 8 June 2011 Accepted: 7 December 2011 Published: 7 December 2011

\section{References}

1. Huygens, C: Oeuvres Completes 1888-1940. Société Hollondaise des Science, Haga.

2. Sandor, J, Bencze, M: On Huygens' trigonometric inequality. RGMIA Res Rep Collect 8(3) (2005). Article 14

3. Zhu, L: A source of inequalities for circular functions. Comput Math Appl. 58, 1998-2004 (2009). doi:10.1016/j. camwa.2009.07.076

4. Neuman, E, Sándor, J: On some inequalities involving trigonometric and hyperbolic functions with emphasis on the Cusa-Huygens, Wilker, and Huygens inequalities. Math Inequal Appl. 13, 715-723 (2010)

5. Mortiti, C: The natural approach of Wilker-Cusa-Huygens inequalities. Math Inequal Appl. 14, 535-541 (2011)

6. Becker, M, Strak, EL: On a hierarchy of quolynomial inequalities for tanx. Univ Beograd Publ Elektrotehn Fak Ser Mat Fiz. , 602-633: 133-138 (1978)

7. Zhu, L, Hua, JK: Sharpening the Becker-Stark inequalities. J Inequal Appl (2010). Article ID 931275

8. Zhu, L: Sharp Becker-Stark-type inequalities for Bessel functions. J Inequal Appl (2010). Article ID 838740

9. Anderson, GD, Qiu, SL, Vamanamurthy, MK, Vuorinen, M: Generalized elliptic integral and modular equations. Pac J Math. 192, 1-37 (2000). doi:10.2140/pjm.2000.192.1 
10. Anderson, GD, Vamanamurthy, MK, Vuorinen, M: Conformal Invariants, Inequalities, and Quasiconformal Maps, New York. (1997)

11. Anderson, GD, Vamanamurthy, MK, Vuorinen, M: Monotonicity of Some Functions in Calculus. Available online at www. math.auckland.ac.nz/Research/Reports/Series/538.pdf

12. Malešević, BJ: One method for proving inequalities by computer. J Ineq Appl (2007). Article ID 78691

doi:10.1186/1029-242X-2011-136

Cite this article as: Chen and Cheung: Sharp Cusa and Becker-Stark inequalities. Journal of Inequalities and Applications 2011 2011:136.

\section{Submit your manuscript to a SpringerOpen ${ }^{\circ}$} journal and benefit from:

- Convenient online submission

- Rigorous peer review

- Immediate publication on acceptance

- Open access: articles freely available online

- High visibility within the field

- Retaining the copyright to your article

Submit your next manuscript at $\gg$ springeropen.com 